\title{
The genus Liparis (Orchidaceae) in Hon Ba nature reserve, Vietnam, Khanh Hoa province
}

\author{
L. V. Averyanov ${ }^{1}$, Truong Ba Vuong ${ }^{2}$, Truong Quang Tam² \\ ${ }^{1}$ Russian Academy of Sciences, Komarov Botanical Institute, Prof. Popov, 2; St. Petersburg, RF-197376, Russia \\ E-mail:av_leonid@mail.ru; av_leonid@yahoo.com \\ ${ }_{2}^{2}$ Institute of Tropical Biology, Department of Biological resources, 85 Tran Quoc Toan St., Distr. 3, Hochiminh City, Vietnam \\ E-mail: bavuong2019@yahoo.com
}

Key words: Orchidaceae, Liparis, Vietnam, Hon Ba nature reserve, plant diversity, plant taxonomy, nature protection.

Summary. Liparis Rich. is rather large genus of orchid family (Orchidaceae), which includes 320-350 species widely distributed in tropical, subtropical and temperate areas of the world with maximal diversity in tropical Asia (Chen et al., 2009). The territory of Eastern Indochina and particularly Vietnam certainly represent one of its richest taxonomic centers. Available modern monographic assessments of the genus in Vietnamese flora record here 49 species belonging to all three its recognized sections (Averyanov, Averyanova, 2003; Averyanov, 2013). Recent field investigations discovered four more species (L. caudata Aver. et K. S. Nguyen, L. delicatula Hook. f., L. gamblei Hook. f., L. longispica Aver. et K. S. Nguyen) new for the flora of Vietnam (Averyanov et al., 2016). In addition, one recently discovered species of the genus is described here (L. honbaensis Aver. et Vuong) and one is recorded as a novelty for the flora of Vietnam ( $L$. tenuis Rolfe ex Downie). As a result, total number of Liparis species recorded in Vietnam reaches presently 55 entities (Table 1). At the same time related floras, such as Thailand, Laos and China comprise 35, 13 and 63 Liparis species respectively, some of which are regarded as local endemics (Seidenfaden, 1976; Newman et al., 2007; Schuiteman et al., 2008; Chen et al., 2009; Tetsana et al., 2013). Some of these species well known in neighboring countries may be found in Vietnam additionally to strictly endemic undescribed species, which undoubtedly inhabit numerous still unexplored mountainous areas of the country. Hence, investigation and inventories of local floras in mountain systems still giving home to rich primary woods are very important to understanding of actual plant diversity of Vietnam. In this connection flora of Hon $\mathrm{Ba}$ Mountains officially protected now as Hon Ba nature reserve represents one of the most significant refuges giving home to aboriginal primary woods harboring many species of ancient migrations and numerous taxa of own local endemism.

\section{Род Liparis (Orchidaceae) в заповеднике Хон Ба, Вьетнам, провинция Кханьхоа}

\author{
Л. В. Аверьянов ${ }^{1}$, Чонг Ба Вуонг², Чонг Куан Там²
}

${ }^{1}$ Ботанический институт им. В.Л. Комарова РАН, ул. Проф. Попова, 2; Санкт-Петербург, 197376, Россия. E-mail:av_leonid@mail.ru; av_leonid@yahoo.com

${ }^{2}$ Институт Тропической Биологии, Отдел биологических ресурсов, 85, ул. Чан Куок Тоан, район 3, Хошимин, Вьетнам E-mail: bavuong2019@yahoo.com

Ключевые слова: Orchidaceae, Liparis, Вьетнам, заповедник Хон Ба, разнообразие растений, таксономия растений, охрана природы. 
Аннотация. Современные ботанические исследования позволяют обозначить Восточный Индокитай и особенно территорию Вьетнама как богатейший мировой центр генетического разнообразия рода Liparis Rich. (Orchidaceae), для которого здесь приводится 56 видов, подтвержденных гербарными экземплярами. Вместе с этим, многие горные области Вьетнама остаются пока слабо исследованными в ботаническом отношении и, без сомнения, скрывают много узкоэндемичных видов, еще не известных науке. Горы Хон Ба, представляющие ныне официально охраняемый заповедник, являются ярким примером такой территории, обладающей исключительно богатой и разнообразной флорой орхидей. Статья представляет первые результаты ботанических исследований гор Хон Ба, проводившихся в течение 2014 года, и содержит современные данные по инвентаризации флоры орхидных, посвященные роду Liparis - одному из крупнейших родов данного семейства во флоре заповедника. Представленный аннотированный обзор включает 13 видов (Liparis sect. Liparis: L. mamillata, L. ngoclinhensis, L. sootenzanensis; L. sect. Cestichis: L. balansae, L. bootanensis, L. cespitosa, L. chapaensis, L. dendrochiloides, L. honbaensis, L. mannii, L. tenuis; L. sect. Distichae: L. compressa, L. gibbosa), найденных на территории заповедника. Для каждого вида приводится правильное название, список важнейших синонимов, цитирование типа, краткое описание, распространение (для эндемиков приводится перечисление местонахождений), данные по экологии, фенологии, а также иллюстрации. Все данные основаны на гербарных экземплярах, помещенных на хранение в LE (Ботанический институт им. В. Л. Комарова РАН), VNM (Музей Института тропической биологии Академии наук Вьетнама) и PSU (Университет принца Сонгкла, Таиланд). Почти все нахождения видов, указанные в статье, являются новыми для территории страны. Один вид (L. honbaensis) описан в качестве нового вида для науки, ещё один (L. tenuis) приводится для Вьетнама впервые, а L. mamillata и L. ngoclinhensis были известны ранее только по типовым образцам, собранным за пределами заповедника.

\section{Introduction}

Liparis Rich. is rather large genus of orchid family (Orchidaceae), which includes 320-350 species widely distributed in tropical, subtropical and temperate areas of the world with maximal diversity in tropical Asia (Chen et al., 2009). The territory of Eastern Indochina and particularly Vietnam certainly represent one of its richest taxonomic centers. Available modern monographic assessments of the genus in Vietnamese flora record here 49 species belonging to all three its recognized sections (Averyanov, Averyanova, 2003; Averyanov, 2013). Recent field investigations discovered four more species (L. caudata Aver. et K. S. Nguyen, L. delicatula Hook. f., L. gamblei Hook. f., L. longispica Aver. et K. S. Nguyen) new for the flora of Vietnam (Averyanov et al., 2016). In addition, one recently discovered species of the genus is described here ( $L$. honbaensis Aver. et Vuong) and one is recorded as a novelty for the flora of Vietnam (L. tenuis Rolfe ex Downie). As a result, total number of Liparis species recorded in Vietnam reaches presently 55 entities (Table 1). At the same time related floras, such as Thailand, Laos and China comprise 35, 13 and 63 Liparis species respectively, some of which are regarded as local endemics (Seidenfaden, 1976; Newman et al., 2007; Schuiteman et al., 2008; Chen et al., 2009; Tetsana et al., 2013). Some of these species well known in neighboring countries may be found in Vietnam additionally to strictly endemic undescribed species, which undoubtedly inhabit numerous still unexplored mountainous areas of the country. Hence, investigation and inventories of local floras in mountain systems still giving home to rich primary woods are very important to understanding of actual plant diversity of Vietnam. In this connection flora of Hon $\mathrm{Ba}$ Mountains officially protected now as Hon Ba nature reserve represents one of the most significant refuges giving home to aboriginal primary woods harboring many species of ancient migrations and numerous taxa of own local endemism.

Hon Ba Mountains represent eastern branch of Dalat Plateau, integrative central part of highland area known in modern Vietnamese geography as Central Highlands, vast elevated area of southern part of Truong Son (Annamite) Range. Various landforms spread here from sea level to the highest point of Hon Ba elevated to $1578 \mathrm{~m}$ a.s.l. Mountainous landscape gives here home to large spectrum of plant communities. Close sea proximity provides very high humidity observed in all elevated highland habitats, which are particularly rich in orchids. There are three new orchid species were found in Hon Ba Mountains recently, namely Vanilla atropogon Shuit., Aver. et Rybkova, Cordiglottis longipedicellata Joongku Lee, T. B. Tran et R. K. Choudhary, and Miguelia cruenta Aver. et Vuong (Choudhary et al., 2013; Schuiteman et al., 2013; Averyanov, Truong Ba Vuong, 2015). Meanwhile, very rich and diverse orchid flora of Hon Ba Mountains remains insufficiently studied. This paper reports results of botanical exploitation conducted in 2014. It contains first modern data of ongoing orchid inventory and is focused on the 
Table 1

\section{Liparis Rich. species hitherto recorded in Vietnam}

Species recorded in Hon Ba Mountains are marked with bold font; species recorded as new for the flora of Vietnam is marked by asterisk (*), species discovered and described as a new for science is marked by double asterisk $(* *)$.

L. sect. Liparis.

L. acuminata Hook. f.

L. acutissima Rchb. f.

L. atrosanguinea Ridl.

L. campylostalix Rchb. f.

L. cordifolia Hook. f.

L. deflexa Hook. f.

L. ferruginea Lindl.

L. gamblei Hook. f.

L. gigantea C. L. Tso

L. longispica Aver. et K. S. Nguyen

L. mamillata Aver.

L. nana Rolfe

L. nervosa (Thunb.) Lindl.

L. ngoclinhensis Aver.

L. odorata (Willd.) Lindl.

L. oppositifolia Szlach.

L. petiolata (D.Don) P. F. Hunt et Summerh.

L. petraea Aver. et Averyanova

L. regnieri Finet

\section{L. sootenzanensis Fukuy \\ L. tschangii Schltr.}

L. sect. Cestichis Lindl.

L. averyanoviana Szlach.

L. balansae Gagnep.

L. bautingensis Tang et F. T. Wang

L. bootanensis Griff.

L. caudata Aver. et K. S. Nguyen

L. cespitosa (Lam.) Lindl.

L. chapaensis Gagnep.

L. delicatula Hook. f.

L. delicatula Hook. f.

L. dendrochiloides Aver.

L. distans C. B. Clarke

L. elliptica Wight

L. emarginata Aver.

L. filiformis Aver.

L. honbaensis Aver. et Vuong**

L. latifolia Lindl.

L. latilabris Rolfe
L. longibracteata Aver.

L. luteola Lindl.

L. mannii Rchb. f.

L. penduliflora Szlach.

L. petelotii Gagnep.

L. plantaginea Lindl.

L. pumila Aver.

L. resupinata $\mathrm{Ridl}$.

L. rhombea J. J. Smith

L. rivularis Aver.

L. sparsiflora Aver.

L. stricklandiana Rchb. f.

L. superposita Ormerod

L. tenuis Rolfe ex Downie*

L. tripartita Aver. et Averyanova

L. viridiflora (Blume) Lindl.

L. sect. 3. Distichae (Ridl.) Aver.

L. compressa (Blume) Lindl.

L. gibbosa Finet genus Liparis, one of the largest orchid genus in the orchid flora of Hon Ba Mountains.

Present illustrated assessment includes annotated list of 13 species discovered in Hon Ba Mountains with their accepted names, main synonyms, data about type, short descriptions, data on distribution (localities in limits of provinces are mentioned only for local Vietnamese endemics), ecology, phenology and illustrations. All presented data are based on collected voucher herbarium specimens listed for each species and housed at LE (Komarov Botanical Institute), VNM (Vietnam museum, Institute of Tropical Biology) and PSU (Prince of Songkla University) Herbaria. Almost all records mentioned in this paper represent new localities of Liparis species in Vietnamese territory. One species (L. honbaensis) is described as a new for science, another (L. tenuis) is recorded for Vietnamese flora for the first time, two species discovered in Hon $\mathrm{Ba}$ Mountains (L. mamillata and L. ngoclinhensis) have been known earlier only from their type localities.

Annotated list of discovered and described species is presented below in a form of standard taxonomic treatment.

\section{Taxonomy treatment \\ Liparis Rich. in Hon Ba Mountains (Hon Ba nature reserve)}

Liparis Rich.

1817, Orch. Europ. Annot.: 21, 30, 38, nom. cons.; Seidenf. 1976, Dansk Bot. Ark. 31, 1: 1-105; Aver., 1994, Identification Guide Viet. Orch.: 136148; id., 2013, Turczaninowia 16, 1: 76-122; Aver. et Averyanova, 2003, Updated Checklist Orch. Viet.: 41-43; Aver. et al., 2015, Wulfenia 22: 162-168.

Type: L. loeselii (L.) Rich. (Ophrys loeselii L.).

The genus includes 3 sections and about 320 species distributed in tropical, subtropical and temperate areas of the world with maximal species diversity in tropical Asia. In Vietnam 56 species are recorded.

$=$ Leptorkis Thouars, 1809, Nouv. Bull. Sci. Soc. Philom. Paris 1: 317.

$=$ Stichorkis Thouars, 1809, 1.c.: 318 .

$=$ Cestichis Thouars, 1822. Hist. Orchid. tab. 90 .

=Disticholiparis Marg. et Szlach., 2004,

Orchidee (Hamburg) 55, 2: 175.

=Platystyliparis Marg., 2006, Richardiana 7, 1: 35 


\section{L. sect. 1. Liparis.}

Aver., 1994, Identification Guide Viet. Orch.: 137-141; id., 2013, Turczaninowia 16, 1: 80-92.

Section includes about 100 species distributed all around generic area with maximal species diversity in tropical Asia. In Vietnam 20 species occur; 3 species are recorded in studied area.

\section{Liparis mamillata Aver.}

2003, in Aver. et Averyanova, 2003, Updated Checklist Orch. Viet.: 82; id., 2013, Turczaninowia 16, 1: 80, Fig. 38e, f, 39c-h.

Type from southern Vietnam ("Dak Lak Prov., Krong Bong Distr., Cu Pui Municipality, elevation from Dak Tour village to main peak of Chu Yang Sinh $\mathrm{mt}$ system, $12^{\circ} 24^{\prime} \mathrm{N}, 108^{\circ} 26^{\prime} \mathrm{E}$, along Dak Tour river, 11 May 2000, Averyanov et al., VH 6046") - LE (holotype), HN (isotype).

Lithophytic herb. Rhizome short, brown, slender, creeping, horizontal. Pseudobulbs 1-leaved, ovoid, $1.6-1.7 \mathrm{~cm}$ high, covered by brownish papyraceous sheaths, densely tufted. Leaf petiolate, glabrous; petiole 1.9-2 cm long; leaf blade ovate, 4.5-4.7 $\mathrm{cm}$ long, 3-3.2 cm wide, acute. Inflorescence synanthous, $10-11 \mathrm{~cm}$ long; peduncle $4.5-5 \mathrm{~cm}$ long, covered by 2 sterile bracts, purplish apically, $0.7-2.5 \mathrm{~cm}$ long; rachis $4.5 \mathrm{~cm}$ long, flattened, with 2 lateral wings about $0.5 \mathrm{~mm}$ wide, 3-7-flowered, pale green from the base to middle, dark purple to the apex; floral bracts ovate, $0.4-0.6 \mathrm{~cm}$ long, acute, dark purple, greenish at apex. Flower about $2 \mathrm{~cm}$ across, sepals pale green, petals and labellum dark purple. Sepals lanceolate, 8-9 mm long, 2.5 $\mathrm{mm}$ wide (being flattened), acute, revolute along the margin. Petals linear filiform, 5-8 $\mathrm{mm}$ long, acute. Labellum simple, ovate, $8-9 \mathrm{~mm}$ long, 4-5 $\mathrm{mm}$ wide, acute, margin dark purple, finely ciliate; base with 2 erect, raising calli coming in form of flat parallel stripes to the labellum apex. Column white, glabrous, 5-6 $\mathrm{mm}$ tall, slender, broadening at the base, apically triangular winged; anther cap pale greenish-yellow, about $1 \mathrm{~mm}$ tall. Pedicel and ovary 5-6 mm long, dark purple, twisted. Fig. 1A, B.

Distribution. Vietnam: Dak Lak (Chu Yang Sinh mt.), Dak Nong (Ta Dung mt.), Khanh Hoa (Hon Ba $\mathrm{mt}$.). Endemic.

Ecology and phenology. Primary humid broadleaved evergreen montane forest at elevation 1400$1500 \mathrm{~m}$. Flowers in May.

Specimen examined. Khanh Hoa province, Hon Ba Mountains, V. B. Truong 113 (VNM).

2. Liparis ngoclinhensis Aver.

2013, Turczaninowia 16, 1: 81, Fig. 38g, 39i-1.

- L. crassibasis auct. non J. J. Sm.: Aver. et
Averyanova, 2003, Updated Checklist Orch. Viet.: 41.

Type from southern Vietnam ("S. Vietnam, Prov. Kontum. ... NW slope of Ngoc Linh mountain system above Long Nam village, L. Averyanov et al., VH 1154") - LE (holotype), HN, MO (isotypes).

Lithophytic herb. Pseudobulbs 1-leaved, close together, ovoid, to $3 \mathrm{~cm}$ tall, covered by thin brownish sheaths. Leaf petiolate; petiole $2-2.5 \mathrm{~cm}$ long; leaf blade sub-orbiculate, 7-8 cm long, 6-6.5 $\mathrm{cm}$ wide, acute or shortly acuminate. Inflorescence synanthous, terminal, 17-19 cm long; peduncle narrowly winged, dark purple, to $9.5 \mathrm{~cm}$ long, covered by sheaths; rachis $8-9 \mathrm{~cm}$ long, dark red, 19-20 flowered; floral bract very small, greenishdark purple, triangular, $1.5-2 \mathrm{~mm}$ long, obtuse. Flowers widely opening; sepals pale green, petals pale purple, labellum purple with pale green. Sepals lanceolate, $1-1.2 \mathrm{~cm}$ long, $3.5 \mathrm{~mm}$ wide, acute, revolute along the margin. Petals linear 8 $\mathrm{mm}$ long, $1.5-2 \mathrm{~mm}$ wide, acute, revolute along the margin. Labellum simple, obovate, $1 \mathrm{~cm}$ long, $1.2 \mathrm{~cm}$ wide, apex emarginate and shortly apiculate at apex, margin finely serrulate; disc with oblong callus coming from base to almost apex of labellum. Column pale yellowish-green, 4-4.5 mm tall, bent at middle, broadening to the base; anther cap pale purple, narrowly ovoid, about $1 \mathrm{~mm}$ long. Pedicel and ovary glabrous, 3.5-4 mm long, dark purple, twisted. Fig. 1C, D.

Distribution. Vietnam: Khanh Hoa (Hon $\mathrm{Ba}$ Mt.), Kon Tum (Ngoc Linh mt.). Endemic.

Ecology and phenology. Primary humid broadleaved evergreen submontane forest at elevation 1100-1200 m. Flowers in August - September.

Specimen examined. Khanh Hoa province, Hon Ba Mountains, V. B. Truong 109 (PSU).

3. Liparis sootenzanensis Fukuy.

1933, Rep. Taihoku Bot. Gard. 3: 84; Su HorngJye, 2000, Fl. Taiwan 5: 950, photo 125; Chen Singchi, Ormerod et Wood, 2009, Fl. China 25: 219; Aver., 2013, Turczaninowia 16, 1: 85, Fig. 42a-g, $43 \mathrm{c}-\mathrm{e}, 44$.

$=$ L. tixieri Guillaum., 1961, Bull. Mus. Hist. Nat. ser. 2, 33: 434.

$\equiv$ Liparis macrantha Hook. f. var. sootenzanensis (Fukuy.) S. S. Ying, 1977, Col. Ill. Indig. Orch. Taiwan 1, 2: 224.

$\equiv$ Liparis nigra Seidenf. var. sootenzanensis (Fukuy.) T. S. Liu et H. J. Su, 1978, Fl. Taiwan 5: 1047.

$=$ L. piriformis Szlach., 1993, Fragm. Florist. Geobot. 38, 2: 456, fig. 5; Aver., 1994, Identification 
Guide Viet. Orch.: 402; Aver. et Averyanova, 2003, Updated Checklist Orch. Viet.: 41.

$=$ L. nigra Seidenf. var. flava Aver., 1999, Bot.

Journ. (St. Petersburg) 84, 10: 128.

$=$ L. Alava (Aver.) Aver., 2003, Updated Checklist Orch. Viet.: 43, 79, fig. 5.

$\equiv$ Diteilis sootenzanensis (Fukuy.) M. A. Clem. et D. L. Jones, 2005, Jones, Orchadian 15: 41.

Type from Taiwan ("N. Fukuyama 4104") KPM (Herb. Orch. Fuk.).

Lithophytic herb. Stems few, arrange close together, fleshy $13-22 \mathrm{~cm}$ long, greenish, slightly curved near base. Leaves 3-4, sessile, sheathed, elliptic, $11-17 \mathrm{~cm}$ long, $4.5-5.8 \mathrm{~cm}$ wide, acuminate, with dark violet veins; leaf sheath, $1.5-2 \mathrm{~cm}$ long. Inflorescence synanthous, terminal, 22-25 cm long, 17-18-flowered; peduncle ridged; floral bracts triangular, 3-5 mm long, acute, purple. Flower widely opening; pale yellowish-green. Dorsal sepal lanceolate, $1.6-2 \mathrm{~cm}$ long, $2 \mathrm{~mm}$ wide, with revolute margin, purplish at apex. Lateral sepals lanceolate, 1.1-1.2 cm long, 2-3 mm wide, acute. Petals linear, slightly curved $1.5-1.6 \mathrm{~cm}$ long, in young flowers reddish-green, red when old. Labellum obovate, about $2 \mathrm{~cm}$ long, emarginated, finely fimbriate; strongly bent at middle; base with 2 triangular calli turning into low rides to the middle of labellum. Column green to yellowish-red. Fig. 1E, F

Distribution. Vietnam: Dak Lak, Dak Nong, Khanh Hoa, Kon Tum, Lam Dong, Nghe An, Quang Binh, Quang Tri, Thua Thien - Hue. Taiwan.

Ecology and phenology. Broad-leaved evergreen submontane forest at elevation 800-900 $\mathrm{m}$, commonly along streams. Flowers in May.

Specimen examined. Khanh Hoa province, Hon Ba Mountains, V.B. Truong 116 (VNM).

Note: This species is very variable according to flower size and color. In Vietnam flower color varies from pure pale yellow-green to dark purple-violet. Flowers in samples observed in studied area were green or pale yellow-green to pale yellow-green with purple.

L. sect. 2. Cestichis Thouars ex Lindl.

1830, Gen. Sp. Orch. Pl.: 29; Aver., 1994, Identification Guide Viet. Orch.: 141-148; id., 2013, Turczaninowia 16, 1: 92-119; Aver. et al., 2015, Wulfenia 22: 162-168. - Cestichis Thouars ex Pfitz. 1887, Entwurf. Naturl. Anord. Orch.: 56; Thouars, 1822, Hist. Orch. Iles Austr. Afr. tab. 90, stat. ambig.

Lectotype: L. cespitosa (Lam.) Lindl. (Epidendrum cespitosum Lam.).

Section includes 100-140 species distributed in tropical areas of the Old World with highest species diversity in SE Asia. In Vietnam 34 species occur; 8 species are recorded in studied area, 1 species is described as a new for science (L. honbaensis).

\section{Liparis balansae Gagnep.}

1932, Bull. Soc. Bot. France 79: 165; id., 1932, Fl. Gen. Indo-Chine 6, 2: 186; Seidenf., 1976, Dansk Bot. Ark., 31, 1: 49, fig. 27; id., 1992, Opera Bot., 114: 138, pl. 9c; Aver., 1994, Identification Guide Viet. Orch.: 146; id., 2013, Turczaninowia 16, 1: 117, Fig. 60c, d; H.H. Pham, 2000, Ill. Fl. Vietnam 3: 901, fig. 11349; Aver. et Averyanova, 2003, Updated Checklist Orch. Viet.: 41; Chen Sing-chi, Ormerod et Wood, 2009, Fl. China 25: 221.

$=$ L. anatina Szlach., 1993, Fragm. Florist. Geobot. 38: 454, fig. 3; Ormerod, 2007, Taiwania 52, 4: 308 .

三Stichorkis balansae (Gagnep.) Marg., Szlach. et Kulak, 2008, Acta Soc. Bot. Poloniae 77: 37.

Described from Vietnam ("Tonkin: ... Bavi ... Cha-pa ... Annam: Mere et l'Enfant, $2000 \mathrm{~m}$ alt., prov. Nhatrang"). Lectotype ("Tonkin, ... Mont Bavi, vers 800 metres, ... 23 Juillet 1886, B. Balansa № 2022") - P [P 00328023], designated here.

Lithophytic herb. Pseudobulb ovoid, about 2 $\mathrm{cm}$ tall, arranged close together, 1-leaved. Leaves sessile, elliptic, to $12 \mathrm{~cm}$ long, $2.8 \mathrm{~cm}$ wide, acute. Inflorescence synanthous, terminal, arching, 20$21.5 \mathrm{~cm}$ long; peduncle winged, with 2 triangular, acuminate bracts, $1.1 \mathrm{~cm}$ long; rachis about $11 \mathrm{~cm}$ long, with 8 distant flowers; floral bract narrowly ovate, $1.5-1.6 \mathrm{~cm}$ long, acuminate. Flowers green, widely opening. Dorsal sepal narrowly lanceolate, $1.3-1.4 \mathrm{~cm}$ long, $3 \mathrm{~mm}$ wide, revolute along the margin, obtuse. Lateral sepals lanceolate, $1-1.2 \mathrm{~cm}$ long, $3 \mathrm{~mm}$ wide, obtuse. Petals linear, $1.2-1.3 \mathrm{~cm}$ long. Labellum broadly obovate, simple, 1.2-1.3 $\mathrm{cm}$ long and wide, emarginate and shortly apiculate at apex, bent near the base; labellum base with 2 short dark green calli, disc with low longitudinal ridge. Column white, green to the apex, 7-8 mm tall, forward curved, winged on apical part; anther cap green, ovoid, 1.5-2 mm tall. Pedicel and ovary slender, 1.3-1.5 cm long, twisted. Fig. 1G, H.

Distribution. Vietnam: Da Nang, Dak Lak, Ha Giang, Ha Tinh, Hanoi, Khanh Hoa, Kon Tum, Lam Dong, Lao Cai, Ninh Thuan, Phu Tho, Thua Thien Hue, Vinh Phuc. China, Thailand.

Ecology and phenology. Broad-leaved evergreen submontane forest at elevation about $1300 \mathrm{~m}$. Flowers in October.

Specimen examined. Khanh Hoa province, Hon Ba Mountains, V. B. Truong 111 (VNM). 


\section{Liparis bootanensis Griff.}

1851, Not. Pl. Asiat. 3: 278; Gagnep., 1932, Fl. Gen. Indo-Chine 6, 2: 183; Seidenf., 1976, Dansk Bot. Ark., 31, 1: 50, fig. 28; id., 1992, Opera Bot., 114: 138; Comber, 1990, Orch. Java: 135, fig.; Seidenf., Wood, 1992, Orch. Malay. Sing.: 233, fig. 98 f-h; Aver., 1994, Identification Guide Viet. Orch.: 145; id., 2013, Turczaninowia 16, 1: 115, Fig. 59, 60a, b; H. H. Pham, 2000, Ill. Fl. Vietnam 3: 901, fig. 11350; Su Horng-Jye, 2000, Fl. Taiwan 5: 938; Pearce et Cribb, 2002, Orch. Bhutan: 204, fig. 49; Aver. et Averyanova, 2003, Updated Checklist Orch. Viet.: 41; Newman et al., 2007, Checkl. Vasc. Pl. Lao: 272; Schuiteman et al., 2008, Nord. Journ. Bot. 26: 295; Chen Sing-chi, Ormerod et Wood, 2009, Fl. China 25: 221; Barretto, Cribb, Gale, 2011, Orch. Hong Kong: 294, fig. 241-244.

三Leptorkis bootanensis (Griff.) Kuntze, 1891, Revis. Gen. P1. 2: 671.

$\equiv$ Stichorkis bootanensis (Griff.) Marg., Szlach. et Kulak, 2008, Acta Soc. Bot. Poloniae 77: 37.

Type from Bhutan ("Bootan, Durunga, "Griffith 1460") - K-LINDL [syntypes - K 000873778, K 000943030].

Epiphytic herb. Pseudobulb 1-leaved, ovoid, to $2 \mathrm{~cm}$ tall, arranged close together. Leaves shortly petiolate; petiole 1-1.2 cm long; leaf blade oblong, $15-16 \mathrm{~cm}$ long, $2.5-3 \mathrm{~cm}$ wide, acute. Inflorescence synanthous, terminal, to $15 \mathrm{~cm}$ long; peduncle winged; rachis to $5 \mathrm{~cm}$ long, arching; floral bracts ovate, 6-10 $\mathrm{mm}$ long, $1.5-2 \mathrm{~mm}$ wide, acuminate. Flowers widely opening, uniform pale greenorange. Dorsal sepal lanceolate, $9 \mathrm{~mm}$ long, $2.5 \mathrm{~mm}$ wide, acute, revolute along margin. Lateral sepals lanceolate, $7 \mathrm{~mm}$ long, $3 \mathrm{~mm}$ wide, acute. Petals linear, about $9 \mathrm{~mm}$ long. Labellum obovate, $8-10$ $\mathrm{mm}$ long, strongly bent near the base, emarginate and shortly apiculate at apex, margin recurved and slightly erose; labellum base with 2 short triangular calli. Column about $6 \mathrm{~mm}$ tall, apex triangular winged; anther cap ovoid, $0.8 \mathrm{~mm}$ tall. Pedicel and ovary about $2 \mathrm{~cm}$ long. Fig. 1I, 2A.

Distribution. Vietnam: Ha Giang, Ha Tinh, Hanoi, Hoa Binh, Khanh Hoa, Kon Tum, Lai Chau, Lam Dong, Lao Cai, Quang Binh, Thai Nguyen, Thanh Hoa, Thu Thien - Hue, Vinh Phuc. Bhutan, China, India, Japan, Java, Malacca Peninsula, Myanmar, Philippines, Taiwan, Thailand.

Ecology and phenology. Primary humid broadleaved evergreen montane forest at elevation 1400-1500 m, usually on mountain tops. Flowers in October.
Specimen examined. Khanh Hoa province, Hon Ba Mountains, P. Rojchana-Umpawan 55, 173 (PSU); V. B. Truong 115 (VNM).

\section{Liparis cespitosa (Lam.) Lindl.}

1825, Bot. Reg. 11, tab 882; Seidenf., 1976, Dansk Bot. Ark., 31, 1: 61, fig. 38; id., 1992, Opera Bot., 114: 139; Comber, 1990, Orch. Java: 135, fig.; id., 2001, Orch. Sumatra: 165; Seidenf., Wood, 1992, Orch. Malay. Sing.: 235, fig. 99 c-e, pl. 13d; Wood, Cribb, 1994, Checklist Orch. Borneo: 90, fig. 6a, b; Aver., 1994, Identification Guide Viet. Orch.: 146; id., 2013, Turczaninowia 16, 1: 108, 53h, i; H. H. Pham, 2000, Ill. Fl. Vietnam 3: 902, fig. 11356; Su Horng-Jye, 2000, Fl. Taiwan 5: 939, fig. 399; Pearce et Cribb, 2002, Orch. Bhutan: 204; Aver. et Averyanova, 2003, Updated Checklist Orch. Viet.: 41; Newman et al., 2007, Checkl. Vasc. Pl. Lao: 272; Schuiteman et al., 2008, Nord. Journ. Bot. 26: 295; Chen Sing-chi, Ormerod et Wood, 2009, Fl. China 25: 222.

E Epidendrum cespitosum Lam., 1783, Encycl. 1: 187.

$\equiv$ Malaxis cespitosa (Lam.) Thouars, 1822, Hist. Orchid.: t. 90.

= Liparis pusilla Ridl., 1886, Journ. Linn. Soc. 22: 294; Gagnep., 1932, Fl. Gen. Indo-Chine 6, 2: 176.

$\equiv$ Leptorkis cespitosa (Lam.) Kuntze, 1891, Revis. Gen. Pl. 2: 671.

$\equiv$ Cestichis cespitosa (Lam.) Ames, 1908, Orchidaceae 2: 132.

= Liparis poilanei Gagnep., 1932, Bull. Soc. Bot. France 79: 166; id., 1932, 1.c. 6, 2: 177, fig. 17, 15-20, 18, 1.

= Liparis mantidopsis Szlach., 1993, Fragm. Florist. Geobot. 38: 459, fig. 7; Aver., 1994, Identification Guide Viet. Orch.: 401; Aver. et Averyanova, 2003, Updated Checklist Orch. Viet.: 42.

$\equiv$ Stichorkis cespitosa (Lam.) Thouars ex Marg. in Marg. et Szlach., 2010, Orchid. Tahiti: 61.

Described from Reunion Island ("Isle De Bourbon"). Type (“Jussieu”) hardily exists. Neotype ("Mauritius, Commerson s.n.") proposed by Pearce et Cribb, 2002, 1.c.: 204, as "lectotype" - P-LA [P 00672996].

Epiphytic herb. Pseudobulbs 1-leaved, ovoid, 5-7 mm tall and wide, arranged close together on short rhizome. Leaves shortly petiolate; petiole 5-6 mm long; leaf blade lanceolate, $1.3-2.25 \mathrm{~cm}$ long, $0.5-0.55 \mathrm{~cm}$ wide, acuminate. Inflorescence synanthous, terminal, glabrous, $3-3.4 \mathrm{~cm}$ long; 
peduncle winged, $1-1.5 \mathrm{~cm}$ long; rachis $1.5-1.7$ cm long, with 6-9 flowers; floral bracts narrowly triangular, 2.5-3 mm long, acute. Flower 3-4 mm across, pale yellow, sepals and petals backward curved. Dorsal sepal oblong, 2-2.5 mm long, 1-1.5 $\mathrm{mm}$ wide, acute. Lateral sepals oblong, $2.5-3 \mathrm{~mm}$ long, $1 \mathrm{~mm}$ wide, acute. Petals linear filiform, 1-2 $\mathrm{mm}$ long. Labellum oblong or somewhat rectangular, $2.5 \mathrm{~mm}$ long, $1 \mathrm{~mm}$ wide, emarginated and shortly apiculate at apex, strongly bent, with 2 short triangular ridges at the base, margin slightly undulate. Column pale yellow, $1.5 \mathrm{~mm}$ tall, slightly forward curved, with narrow wing at apex; anther cap pale yellow, about $0.4 \mathrm{~mm}$ tall, ovoid or halfglobular. Pedicel and ovary slender, 3-5 mm long. Capsule when young ovoid, $3-3.5 \mathrm{~mm}$ long. Fig. 2B, C.

Distribution. Vietnam: Dien Bien, Dong Nai, Ha Giang, Hoa Binh, Khanh Hoa, Kon Tum, Lam Dong, Lao Cai, Ninh Thuan. Tropical area of the Old World extending to islands of western Pacific.

Ecology and phenology. Primary humid broadleaved evergreen submontane forest at elevations 900-1000 m, commonly along streams. Flowers in September.

Specimen examined. Khanh Hoa province, Hon Ba Mountains, P. Rojchana-Umpawan 274 (PSU), V. B. Truong 107 (VNM).

\section{Liparis chapaensis Gagnep.}

1932, Bull. Soc. Bot. France 79: 166; id., 1932, Fl. Gen. Indo-Chine 6, 2: 186, fig. 18, 2-7; Seidenf., 1976, Dansk Bot. Ark., 31, 1: 58, fig. 35; id., 1992, Opera Bot., 114: 138, fig. 83; Aver., 1994, Identification Guide Viet. Orch.: 146; id., 2013, Turczaninowia 16, 1: 114, Fig. 56h, i; H. H. Pham, 2000, Ill. Fl. Vietnam 3: 901, fig. 11352; Aver. et Averyanova, 2003, Updated Checklist Orch. Viet.: 41; Newman et al., 2007, Checkl. Vasc. P1. Lao: 272; Chen Singchi, Ormerod et Wood, 2009, Fl. China 25: 223.

$\equiv$ Stichorkis chapaensis (Gagnep.) Marg., Szlach. et Kulak, 2008, Acta Soc. Bot. Poloniae 77: 37.

Type from north-western Vietnam ("Tonkin... Lo-qui-ho, pres Cha-pa, 1800 m, Septembre 1929 Petelot 5173") - P [P 00327840].

Lithophytic herb. Pseudobulbs 1-leaved, ovoid, to $2 \mathrm{~cm}$ tall, $0.6-1 \mathrm{~cm}$ wide, densely arranged on short rhizome. Leaves shortly petiolate; petiole about $5 \mathrm{~mm}$ long; leaf blade narrowly oblanceolate, 4.5-6 cm long, 7.5-8 $\mathrm{mm}$ wide, acuminate. Inflorescence synanthous, terminal, arching, 4-4.8 $\mathrm{cm}$ long; peduncle about $2.8 \mathrm{~cm}$ long, slightly flattened, winged, wings about $0.5 \mathrm{~mm}$ long; rachis about $2 \mathrm{~cm}$ long; floral bracts subulate narrowly lanceolate, $3.5-5.5 \mathrm{~mm}$ long, $1 \mathrm{~mm}$ wide, acute to acuminate. Flower widely opening, sepals and petal greenish-yellow, labellum greenish-yellow to pale orange. Dorsal sepal linear, about $5 \mathrm{~mm}$ long, $1.5-2 \mathrm{~mm}$ wide, acute, revolute along margin. Lateral sepals linear, about $7 \mathrm{~mm}$ long, $2 \mathrm{~mm}$ wide, acute. Petals linear filiform, 5-6 mm long. Labellum simple, obovate, $6.5 \mathrm{~mm}$ long, $5 \mathrm{~mm}$, truncate and shortly mucronate at apex, strongly bent at middle; margin slightly denticulate; with 2 triangular calli at base. Column white, $4-4.5 \mathrm{~cm}$ tall, curved forward, with short triangular wings at apex. Pedicel and vary green, slender, about $6 \mathrm{~mm}$ long. Fig. 2D, E.

Distribution. Vietnam: Ha Giang, Ha Tinh, Khanh Hoa, Lam Dong, Lao Cai, Ninh Binh, Vinh Phuc. China, Myanmar.

Ecology and phenology. Primary humid broadleaved evergreen montane forest at elevation about $1500 \mathrm{~m}$, commonly along small streams. Flowers in July.

Specimen examined. Khanh Hoa province, Hon Ba Mountains, V. B. Truong 106 (VNM)

\section{Liparis dendrochiloides Seidenf. ex Aver.}

1988, Bot. Journ. (Leningrad) 73, 3: 432; id., 1994, Identification Guide Viet. Orch.: 145; id., 2013, Turczaninowia 16, 1: 112, Fig. 56c, d, 57a; Seidenf., 1992, Opera Bot., 114: 139, fig. 84; H. H. Pham, 2000, Ill. Fl. Vietnam 3: 902, fig. 11354; Aver. et Averyanova, 2003, Updated Checklist Orch. Viet.: 42; Schuiteman et al., 2008, Nord. Journ. Bot. 26: 296.

三Stichorkis dendrochiloides (Seidenf. ex Aver.) Marg., Szlach. et Kulak, 2008, Acta Soc. Bot. Poloniae $77: 37$.

- Liparis dendrochilum auct. non Rchb. f.: Gagnep., 1932, Fl. Gen. Indo-Chine 6, 2: 175.

Type from southern Vietnam ("Annam, 12 Decembre 1912, Eberhardt 58B”) - P [P 00338438].

Epiphytic herb. Pseudobulbs 1-leaved, ovoid, about $2 \mathrm{~cm}$ tall, arranged close together. Leaves shortly petiolate; petiole $1.4-1.5 \mathrm{~mm}$ long; leaf blade lanceolate, $8-10 \mathrm{~cm}$ long, $1.5-1.8 \mathrm{~cm}$ wide, acute. Inflorescence synanthous, terminal, $15-18 \mathrm{~cm}$ long; peduncle longitudinally ridged, at base with 5 acuminate bracts $0.7-2.5 \mathrm{~cm}$ long, 5-8 $\mathrm{mm}$ wide, in middle part with 1 sterile, triangular, acuminate bract about $8 \mathrm{~mm}$ long; rachis about $5 \mathrm{~cm}$ long; floral bracts narrowly triangular, acuminate, about $5 \mathrm{~mm}$ long, longer than flower. Flower pale yellow, small, widely opening. Sepals narrowly ovate, 
2-2.5 mm long, $0.5-1 \mathrm{~mm}$ wide, acute, apically recurved. Petals linear filiform, 1.5-2 mm long, revolute along margin. Labellum simple, obovate, 2-2.5 $\mathrm{mm}$ long, $2 \mathrm{~mm}$ wide, acute, strongly bent at the base, channeled, acute. Column about $1.5 \mathrm{~mm}$ tall, slightly curved at middle. Capsule sub-globular, longitudinally ridged. Fig. 2F, G.

Distribution. Vietnam: Khanh Hoa (Hon Ba mt.), Lam Dong, Lao Cai (sine loc.), Quang Binh (Bo Trach; Le Thuy; Quang Ninh), Thanh Hoa (Ba Thuoc), Thua Thien - Hue (Phu Loc; Xuan Loc). Endemic.

Ecology and phenology. Broad-leaved evergreen humid montane forest at elevation about $1500 \mathrm{~m}$, commonly on mountain tops. Flowers in July.

Specimen examined. Khanh Hoa province, Hon Ba Mountains, V.B. Truong 117 (VNM).

Note. This species is very similar to L. longibracteata Aver., but well differs in leaves shape and pseudobulbs arrangement.

\section{Liparis honbaensis Aver. et Vuong, sp. nov.}

Type from Southern Vietnam ("Khanh Hoa province, Hon Ba nature reserve, Hon Ba Mountain, evergreen mountain forest, lithophyte on mossy dry rocks at elevation about $1410 \mathrm{~m}$ a.s.1., October 2015, Truong Ba Vuong, BV 118 / AL 90") - LE (holotype).

Sympodial lithophytic herb. Pseudobulbs densely clustering, touching each other, narrowly ovoid to ovoid, (1.2)1.5-2(2.2) cm long, (0.8)1$1.5(1.8) \mathrm{cm}$ in diam. Leaves 2, sessile, oblanceolate, (6)8-14(16) cm long, (0.8)1-1.2(1.4) cm wide, acute, with prominent median vein. Inflorescence synanthous, erect, subdense raceme, (15)16$22(25) \mathrm{cm}$ long; scape terete, wingless, or with hardly visible wings, (8)10-15(17) $\mathrm{cm}$ long, with 1-2(3) acute sterile bracts, (1)1.5-3(4.5) cm long, (0.5)0.8-1.5(2) mm wide, rachis (6)7-12(15) $\mathrm{cm}$ long, (20)25-35 flowered. Floral bracts acute, narrowly triangular lanceolate, (4)5-10(12) cm long, (0.6)1-1.4(1.8) mm wide, as long as pedicel and ovary, or slightly longer. Flower pale yellowishgreen, widely opening, $4-5 \mathrm{~mm}$ across. Sepals broadly lanceolate, 4-5 $\mathrm{mm}$ long, $0.8-1.2 \mathrm{~mm}$ wide, obtuse, usually strongly recurved, revolute along the margin; median sepal often cucullate. Petals linear to filiform, straight, slender, backward directed, 5-6 $\mathrm{mm}$ long, flattened $0.3-0.4 \mathrm{~mm}$ wide, revolute along the margin. Labellum oblong obovate, $4-5 \mathrm{~mm}$ long, 1.8-2.2 $\mathrm{mm}$ wide, at the base with low twin callus, strongly curved and laterally inflated near the middle; epichile half-round to sub-quadrate,
1.8-2.2 $\mathrm{mm}$ long and wide, round to truncate, finely irregularly erose along margin, sometimes with small obscure medial dent. Column slender, slightly curved, simple, wingless, 2.5-3(3.2) mm long, slightly broadening to the base, $0.5-0.6 \mathrm{~mm}$ in diam.; anther cap white, broadly ovoid, truncate at front, $0.5-0.6 \mathrm{~mm}$ across. Pedicel and ovary green, terete, shallowly grooved, (5)6-8(10) mm long, $0.8-1 \mathrm{~mm}$ in diam. Fig. 2H, 3, 4A-D.

Distribution. Vietnam: Khanh Hoa (Hon $\mathrm{Ba}$ Mt.). Endemic.

Ecology and phenology. Primary humid broadleaved evergreen montane forest at elevation 1400-1450 m. Flowers in October - November.

Note. New species was found as a rather common plant growing on tops of large mossy boulders together with Oberonia longibracteata Lindl. and Eria gagnepainii A. D. Hawkes et A. H. Heller in rather dry conditions. Among known species it is most close to L. luteola Lindl., from which dramatically differs in erect, dense, many-flowered inflorescence and two prominent lateral swellings on sides of hypochile.

\section{Liparis mannii Rchb. f.}

1872, Flora 55: 275; Gagnep., 1932, Fl. Gen. Indo-Chine 6, 2: 175; Seidenf., 1976, Dansk Bot. Ark., 31, 1: 60, fig. 36; id., 1992, Opera Bot., 114: 138; Aver., 1994, Identification Guide Viet. Orch.: 147; id., 2013, Turczaninowia 16, 1: 104, Fig. 51g, h; H. H. Pham, 2000, Ill. Fl. Vietnam 3: 902, fig. 11355; Pearce et Cribb, 2002, Orch. Bhutan: 208; Aver. et Averyanova, 2003, Updated Checklist Orch. Viet.: 42; Chen Sing-chi, Ormerod et Wood, 2009, Fl. China 25: 224.

三Leptorkis mannii (Rchb. f.) Kuntze, 1891, Revis. Gen. Pl. 2: 671.

$\equiv$ Stichorkis mannii (Rchb. f.) Marg., Szlach. et Kulak, 2008, Acta Soc. Bot. Poloniae 77: 38.

Type from nort-eastern India ("Assam, Mann 27, Herb. Reichenbach 46235”) - W, G [G 00354689 isotype].

Epiphytic herb. Pseudobulbs 1-leaved, arranged close together, ovoid, $1.5-2 \mathrm{~cm}$ tall. Leaves lanceolate, 9-17 cm long, 0.6-0.8 cm wide, acute. Inflorescence synanthous, terminal, $12-20 \mathrm{~cm}$ long; peduncle winged, with 2 sterile, white, linear bracts 8-9.5 mm long; rachis 6-10 $\mathrm{mm}$ long; floral bract linear, $2.5-3 \mathrm{~mm}$ long. Flower pale yellow, widely opening. Sepals, lanceolate, 2.5-3.5 $\mathrm{mm}$ long, obtuse, revolute along margin. Petals filiform, as long as sepals. Labellum 3-3.5 mm long, 3-lobed; median lobe somewhat rhomboid, $3 \mathrm{~mm}$ long, 2-2.5 
$\mathrm{mm}$ wide, margin irregularly erose; side lobes erect, falcate, 1.5-2 mm long, acute. Column erect, about $2 \mathrm{~mm}$ long, broadening at the base, curved forward; anther cap pale yellow, ovoid, about $1 \mathrm{~mm}$ tall. Pedicel and ovary 3-3.5 mm long. Fig. 4E-H.

Distribution. Vietnam: Bac Kan, Cao Bang, Dak Lak, Dien Bien, Ha Giang, Hoa Binh, Khanh Hoa, Lam Dong, Lang Son, Ninh Binh, Phu Tho, Quang Binh, Son La, Thai Nguyen, Thanh Hoa, Tuyen Quang. Bhutan, China, India.

Ecology and phenology. Humid broad-leaved evergreen dense montane forest at elevation about $1500 \mathrm{~m}$, commonly on mountain tops. Flowers in October.

Specimen examined. Khanh Hoa province, Hon Ba Mountains, V. B. Truong 114 (VNM).

\section{Liparis tenuis Rolfe ex Downie}

1925, Bull. Misc. Inform. Kew 1925: 372; Gagnep., 1932, Fl. Gen. Indo-Chine 6, 2: 187; Seidenf., 1976, Dansk Bot. Ark., 31, 1: 94, fig. 64; Aver., 2005, Turczaninowia 8, 1: 76, tab. 2a; id., 2013, Turczaninowia 16, 1: 102, Fig. 52k-n.

$\equiv$ Platystyliparis tenuis (Rolfe ex Downie) Marg., 2007, Richardiana 7: 40.

Type from north-western Thailand ("Doi Suthep, 3200 ft., Kerr 250") - K (syntypes - K 000596220 , K 000596221).

Epiphytic herb. Pseudobulbs 2-leaved, narrowly ovoid, $1.15-1.2 \mathrm{~cm}$ tall, $4-5.5 \mathrm{~mm}$ wide, with $1-2$ longitudinal ridges. Leaves $2-3 \mathrm{~cm}$ long, $7-8 \mathrm{~mm}$ wide, acute. Inflorescence heteranthous, terminal, $7.2-7.5 \mathrm{~cm}$ long; peduncle with 5 , ovate, acute bracts 3-3.5 mm long; rachis 4-4.1 cm long, bearing to 8 flowers; floral bracts narrowly ovate, acute, 2-3 mm long. Flower widely opening, pale green. Sepals narrowly lanceolate to lanceolate, 4-5.5 $\mathrm{mm}$ long, 1-2 $\mathrm{mm}$ wide, acute, revolute along the margin. Labellum glossy, $2.5 \mathrm{~mm}$ long, $2.5-3 \mathrm{~mm}$ wide, obtuse, shortly apiculate at middle; strongly recurved, epichile $2 \mathrm{~mm}$ long, $2.5 \mathrm{~mm}$ wide, suborbicular; hypochile claw-shaped, disk with 2 triangular ridges. Column 3-4 mm long; anther cap ovoid, about $0.7 \mathrm{~mm}$ tall, acute. Pedicel and ovary 3-3.5 mm long. Fig. 4I, 5A-C.

Distribution. Vietnam: Khanh Hoa. Thailand.

Ecology and phenology. Broad-leaved, evergreen forest, commonly along streams. Flowers in September.

Specimen examined. Khanh Hoa province, Hon Ba Mountains, V. B. Truong 108 (VNM).

Note. This is very rare species found in Vietnam for the first time. Further studies of Hon Ba specimens are necessary to compare them with closely related L. delicatula Hook. f. recently reported from neighbor area in Khanh Hoa province, Khanh Son district (Averyanov et al., 2015).

L. sect. 3. Distichae (Ridl.) Seidenf. ex Aver.

1994, Identification Guide Viet. Orch.: 148; id., 2013, Turczaninowia 16, 1: 119-122. - L. grex Distichae Ridl., 1886, Journ. Linn. Soc. London (Bot.) 22: 258, 291.

Lectotype: $L$. disticha Lindl.

Section includes about 10 species distributed in mainland tropical Asia and Indonesia. In Vietnam and in studied area 2 species occur.

12. Liparis compressa (Blume) Lindl.

1830, Gen. Sp. Orchid. P1.: 32; Comber, 1990, Orch. Java: 133, fig.; id., 2001, Orch. Sumatra: 147, fig.; Seidenf. et Wood, 1992, Orch. Pen. Mal. Sing.: 239, fig. 102 d-f; Wood et Cribb, 1994, Checklist Orch. Borneo: 90, fig. 5c, d; Aver. et Averyanova, 2003, Updated Checklist Orch. Viet.: 41; Aver., 2012, Turczaninowia 15, 1: 12, Fig. 1, 1-o; id., 2013, Turczaninowia 16, 1: 119, Fig. 60h, i.

= Malaxis compressa Blume, 1825, Bijdr.: 390.

$\equiv$ Leptorkis compressa (Blume) Kuntze, 1891, Revis. Gen. P1. 2: 671.

$\equiv$ Cestichis compressa (Blume) Ames, 1905, Orchidaceae 1: 8 .

$\equiv$ Disticholiparis compressa (Blume) Marg. et Szlach., 2004, Orchidee (Hamburg) 55: 176.

$\equiv$ Stichorkis compressa (Blume) J. J. Wood, 2011, Orchids Mount Kinabalu 2: 531.

Type from Java ("Crescit: in sylvis altioribus Provinciarum Buitenzorg, Bantam et Tjanjor, Blume s.n.") - BO?

Epiphytic herb. Rhizome slender, creeping, about $3 \mathrm{~mm}$ in diam., covered by brownish, papyraceous scales. Pseudobulbs 1-leaved, ovoid, $1.3-1.5 \mathrm{~cm}$ tall, distant on rhizome on $2.3-4 \mathrm{~cm}$. Leaves shortly petiolate; petiole $1.3-2.5 \mathrm{~cm}$ long; leaf blade narrowly lanceolate, $19-23 \mathrm{~cm}$ long, $1.5-1.8 \mathrm{~cm}$ wide, acute, tapering to the base. Inflorescences heteranthous, terminal, $15-25 \mathrm{~cm}$ long, laterally compressed, winged; rachis about $2.5 \mathrm{~cm}$ long with dense, distichous, persistent, conduplicate floral bracts; floral bracts 5-7 mm long, 2 lowest bracts distinctly larger, rising continuously with the inflorescence wings. Flowers opening in succession by 1 or 2 in same time, widely opening, pale orange, about $1.3 \mathrm{~cm}$ across, sepals curved behind labellum, petals directed at $90^{\circ}$ to ovary. Sepals lanceolate, $1-1.1 \mathrm{~cm}$ long, about $2 \mathrm{~mm}$ wide, slightly revolute 


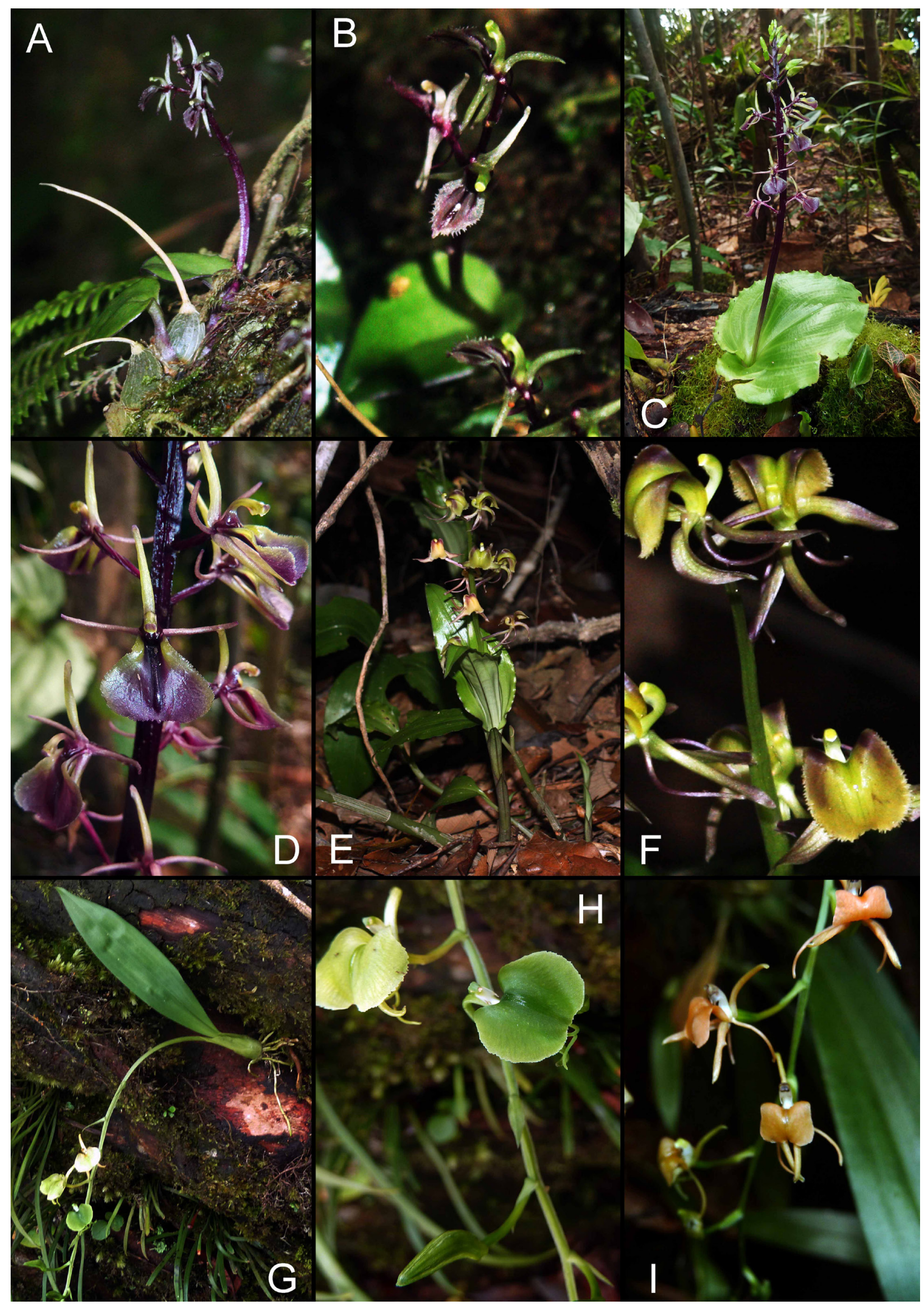

Fig. 1. Liparis in Hon Ba nature reserve. A, B - L. mamillata Aver. (V. B. Truong 113). C, D - L. ngoclinhensis Aver. (V. B. Truong 109). E, F -L. sootenzanensis Fukuy. (V. B. Truong 116). G, H - L. balansae Gagnep. (V. B. Truong 111). I - L. bootanensis Griff. (V. B. Truong 115). All photos by Truong Ba Vuong, correction and design by L. Averyanov. 


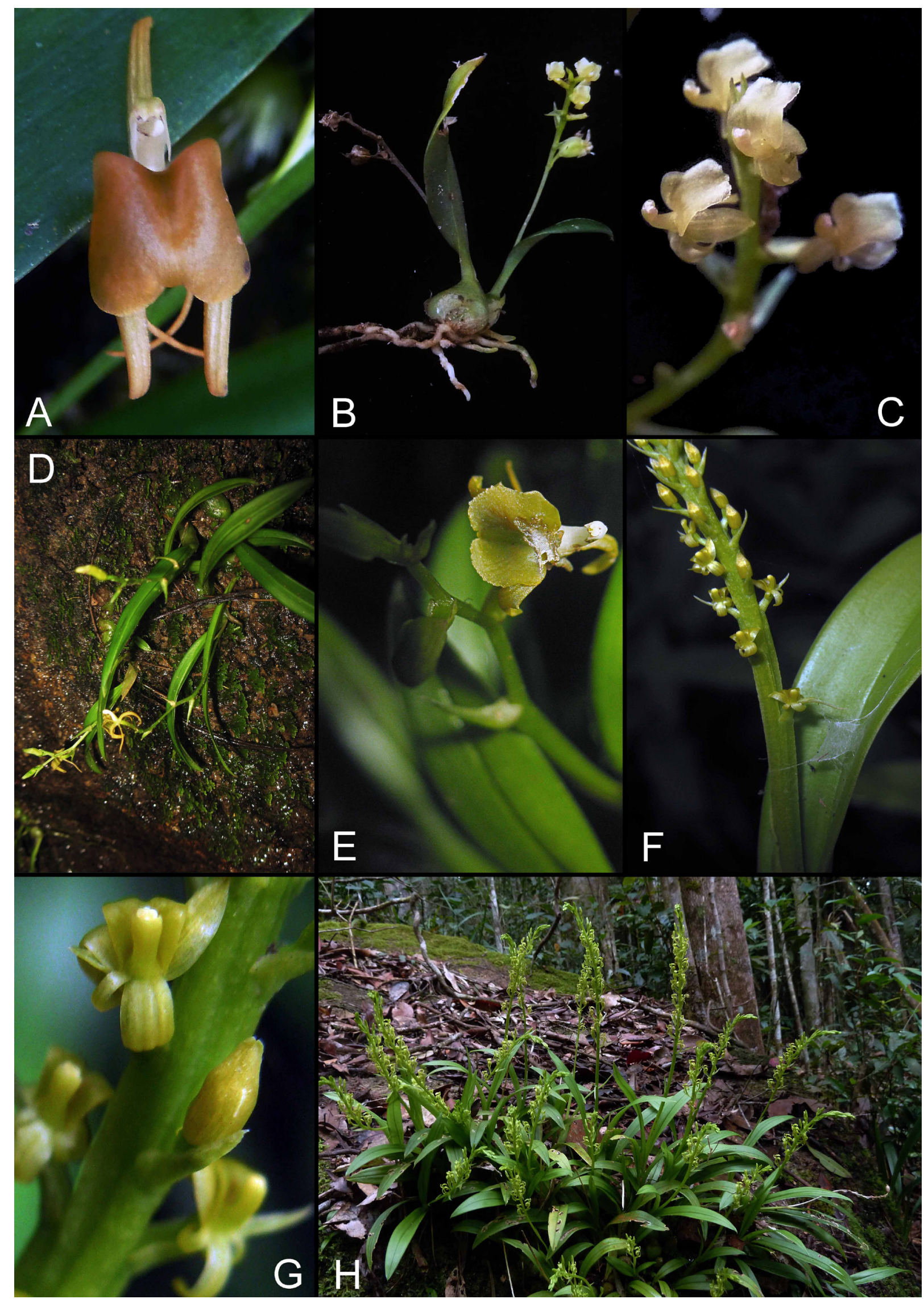

Fig. 2. Liparis in Hon Ba nature reserve. A - L. bootanensis Griff. (V. B. Truong 115). B, C - L. cespitosa (Lam.) Lindl. (V. B. Truong 107). D, E - L. chapaensis Gagnep. (V. B. Truong 106). F, G - L. dendrochiloides Seidenf. ex Aver. (V. B. Truong 117). H - L. honbaensis Aver. et Vuong (type, Truong Ba Vuong, BV $118 / A L$ 90). All photos by Truong Ba Vuong, correction and design by L. Averyanov. 


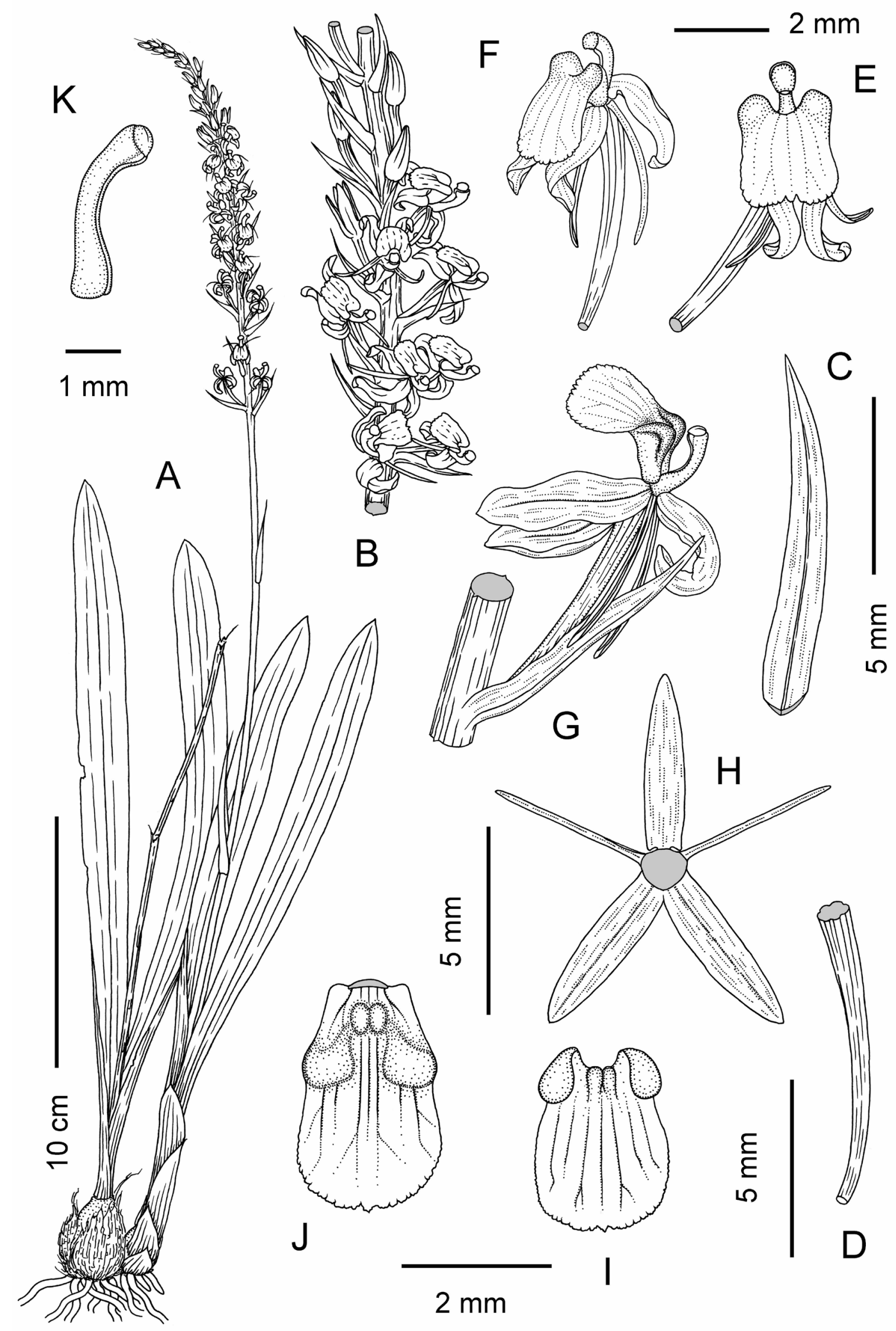

Fig. 3. Liparis in Hon Ba nature reserve. L. honbaensis Aver. et Vuong. A-Flowering plant. B - Portion of inflorescence. C - Floral bract. D - Pedicel and ovary. E, F - Flower, frontal and half side views. G - Flower, floral bract, pedicel, ovary and portion of rachis, side view. $\mathrm{H}$ - Flattened sepals and petals. I - Intact lip, frontal view. J - Flattened lip. $\mathrm{K}$ - Column, side view. All drawn from the type - Truong Ba Vuong, BV 118 / AL 90 by L. Averyanov and T. Maisak. 


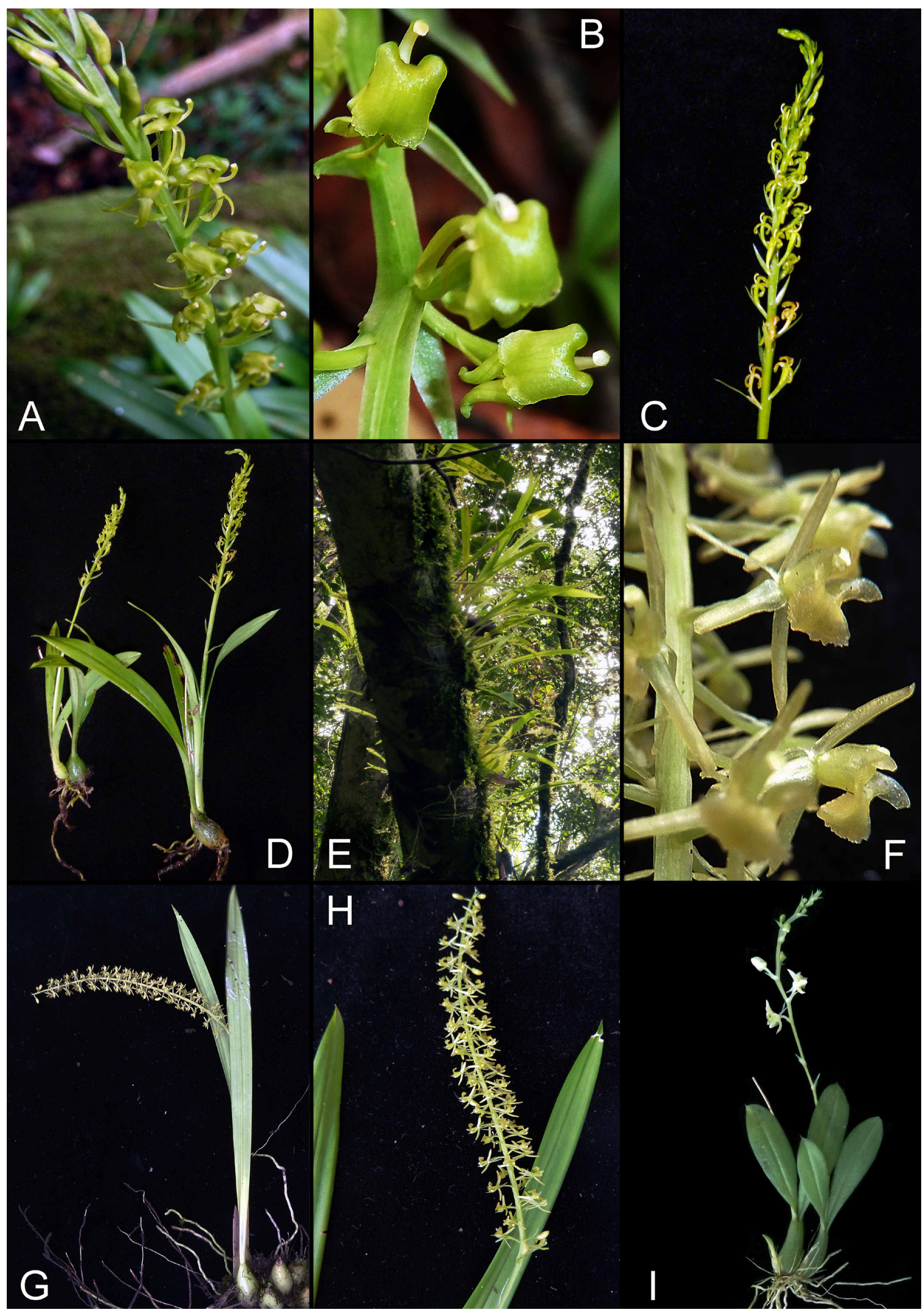

Fig. 4. Liparis in Hon Ba nature reserve. A-D - L. honbaensis Aver. et Vuong (type, Truong Ba Vuong, BV 118 / AL 90). E-H - Liparis mannii Rchb.f. (V.B. Truong 114). I - Liparis tenuis Rolfe ex Downie (V. B. Truong 108). All photos by Truong Ba Vuong, correction and design by L. Averyanov. 


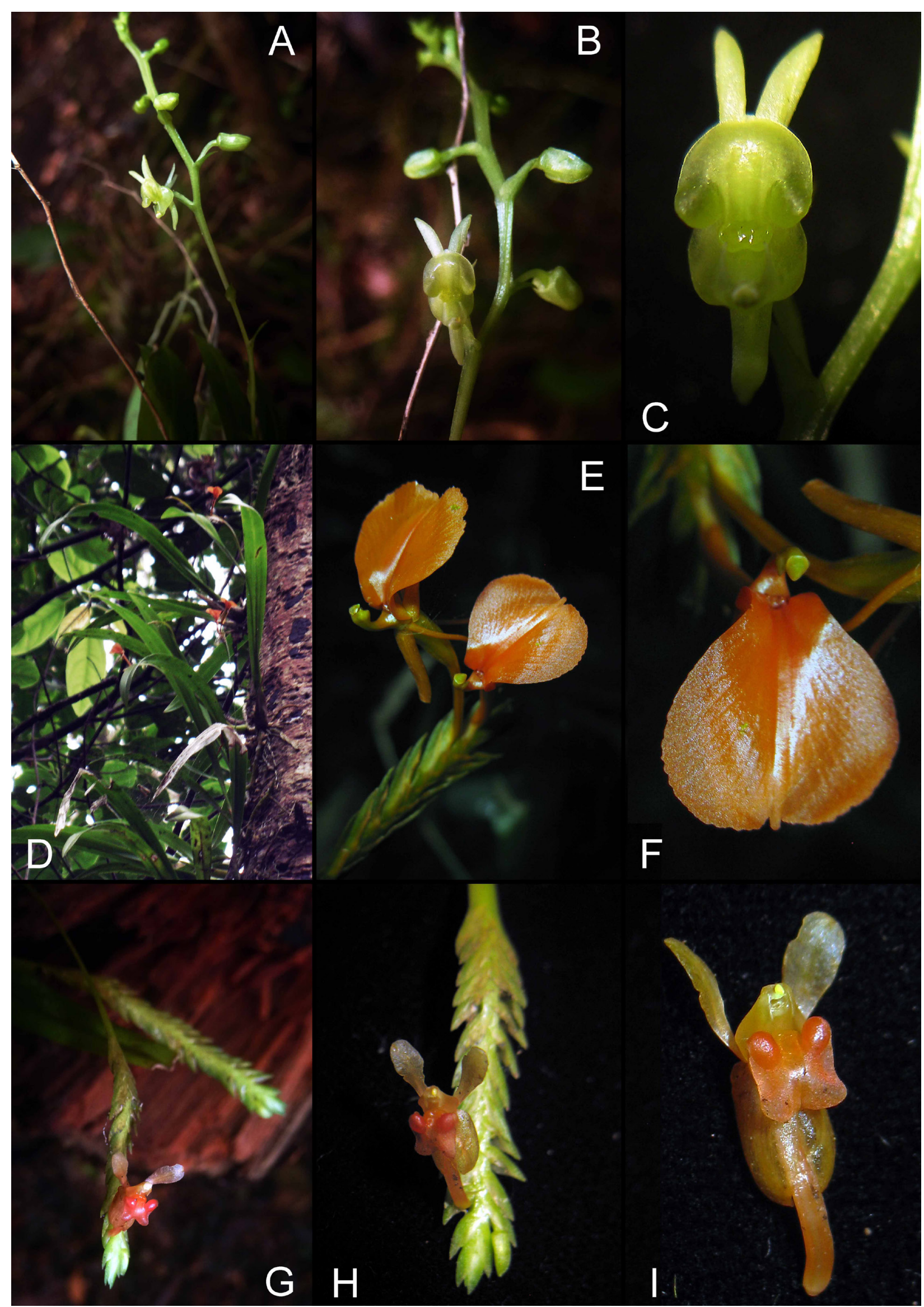

Fig. 5. Liparis in Hon Ba nature reserve. A-C - Liparis tenuis Rolfe ex Downie (V. B. Truong 108). D-F - Liparis compressa (Blume) J. J. Wood (V. B. Truong 109). G-I - Liparis gibbosa Finet (V. B. Truong 110). All photos by Truong Ba Vuong, correction and design by L. Averyanov. 
along the margin, dorsally keeled. Petals linear, about $1 \mathrm{~cm}$ long, acute, with revolute margin. Labellum orbicular, 1.1-1.2 cm long and wide, emarginated and shortly mucronate at apex; shortly clawed at the base, strongly bent; disc with median ridge coming from the base to the lip apex. Column about $5 \mathrm{~mm}$ tall, broadening at the base, strongly forward curved; anther cap yellow-green, ovoid, about $1 \mathrm{~mm}$ tall. Pedicel and ovary orange, slender, $1.2-1.3 \mathrm{~cm}$ long. Fig. 5D-F.

Distribution. Vietnam: Dak Lak, Khanh Hoa, Lam Dong. Java, Kalimantan, Malacca Peninsula, Philippines, Sulawesi, Sumatra.

Ecology and phenology. Primary dense evergreen broad-leaved and mixed submontane forest at elevation 1300-1350 m, commonly along streams. Flowers in July.

Specimen examined. Khanh Hoa province, Hon Ba Mountains, V. B. Truong 109 (VNM).

\section{Liparis gibbosa Finet}

1908, Bull. Soc. Bot. France 55: 342; Seidenf., 1976, Dansk Bot. Ark., 31, 1: 95, fig. 65; id., 1992, Opera Bot., 114: 145; Comber, 1990, Orch. Java: 133, fig.; id., 2001, Orch. Sumatra: 148, fig.; Seidenf. et Wood, 1992, Orch. Malay. Sing.: 240, fig. 102 g-i, pl. 14d; Wood et Cribb, 1994, Checklist Orch. Borneo: 93, fig. 5e, f; Aver., 1994, Identification Guide Viet. Orch.: 148; id., 2013, Turczaninowia 16, 1: 119, Fig. 61a, b; H. H. Pham, 2000, Ill. Fl. Vietnam 3: 909, fig. 11382; Pearce et Cribb, 2002, Orch. Bhutan: 212; Aver. et Averyanova, 2003, Updated Checklist Orch. Viet.: 42; Newman et al., 2007, Checkl. Vasc. Pl. Lao: 272; Schuiteman et al., 2008, Nord. Journ. Bot. 26: 296.

$\equiv$ Disticholiparis gibbosa (Finet) Marg. et Szlach., 2004, Orchidee (Hamburg) 55: 178.

$\equiv$ Stichorkis gibbosa (Finet) J. J. Wood, 2011, Orchids Mount Kinabalu 2: 531.

Type from Java (“Java, s.n.") - P [P 00360796].

Epiphytic herb. Rhizome slender, creeping, 2-3 $\mathrm{mm}$ in diam., many nodal. Pseudobulbs 1-leaved, ovoid, slightly flattened, $1-1.2 \mathrm{~cm}$ tall, distant on rhizome on $0.5-2 \mathrm{~cm}$. Leaves shortly petiolate; petiole $2-2.3 \mathrm{~cm}$ long; leaf blade narrowly lanceolate, $15-18 \mathrm{~cm}$ long, $8-9 \mathrm{~mm}$ wide, acute. Inflorescence synanthous, terminal, $13-15 \mathrm{~cm}$ long; peduncle flat, winged, sterile bracts $1.5 \mathrm{~mm}$ long; rachis $2.5-3.5 \mathrm{~cm}$ long, with dense distichous, persistent, conduplicate floral bracts; floral bracts 5-6 $\mathrm{mm}$ long, $1.5 \mathrm{~mm}$ wide, acute. Flower orange, about $6 \mathrm{~mm}$ across, sepals curved down, petals erect. Sepals narrowly ovate, 4-5 mm long, $2 \mathrm{~mm}$ wide, obtuse, slightly revolute along the margin, with 1 orange vein. Petals oblanceolate, $4 \mathrm{~mm}$ long, $1.5 \mathrm{~mm}$ wide, obtuse. Labellum 3-3.5 mm long, acute, strongly recurved and shortly clawed, disc laterally gibbous, forming 2 rounded calli, with 2 small fleshy ridges. Column $3 \mathrm{~mm}$ tall, winged at apex; anther cap glabrous, broadly ovoid, about 0.9 $\mathrm{mm}$ tall. Pedicel and ovary orange, slender, 5-7 mm long. Capsule obovoid, about $7 \mathrm{~mm}$ long. Fig. 5G-I.

Distribution. Vietnam: Khanh Hoa, Lam Dong, Ninh Thuan. Indonesia, Laos, Malacca. Peninsula, Myanmar, New Guinea, Sri Lanka, Thailand, islands of western Pacific.

Ecology and phenology. Primary dense evergreen broad-leaved and mixed submontane forest at elevation 1000-1050 m, commonly along streams. Flowers in September.

Specimen examined. Khanh Hoa province, Hon Ba Mountains, V. B. Truong 110 (VNM).

\section{Acknowledgements}

Laboratory works, results of which are presented in this paper were financially supported in part by Russian Foundation for Basic Research ("Plant taxonomy, geography and biology in local floras of eastern Indochina"), 15-04-00419A, and were carried out in the framework of institutional research project of the Komarov Botanical Institute of the Russian Academy of Sciences. Field explorations were funded by the Conservation and Research Foundation (U.S.A.).

\section{REFERENCES}

Averyanov L. V. 2013. The orchids of Vietnam illustrated survey. Part 4. Subfamily Epidendroideae. Turczaninowia 16, 1: 5-163.

Averyanov L. V., Averyanova A. L. 2003. Updated Checklist of the Orchids of Vietnam. Vietnam National University Publishing Hose, Hanoi, pp. 101.

Averyanov L.V., Khanh Sinh Nguyen, Nguyen Thien Tich, Phi Tam Nguyen, Van Duy Nong, Van Canh Nguyen, Canh Chu Xuan. 2016. New orchids in the flora of Vietnam. Wulfenia 22: 137-188.

Averyanov L. V., Truong Ba Vuong. 2015. Review of the genus Miguelia (Orchidaceae) with a new species, M. cruenta, from southern Vietnam. Taiwania 60 (1): 33-38. DOI: 10.6165/tai.2015.60.1.33. 
Chen S. C., Ormerod P., Wood J. J. 2009. Liparis Rich. In: Wu Z. Y., Raven P. H., Hong D. Y. (Eds). Flora of China Vol. 25. Science Press, Beijing; Missouri Botanical Garden Press, St. Louis, pp. 211-228.

Choudhary R. K., Tran The Bach, Do Van Hai, Bui Hong Quang, Luu Van Nong, Pankaj Kumar, SangHong Park, Joongku Lee. 2013. Cordiglottis longipedicellata (Orchidaceae), a new species from Vietnam. Annales Botanici Fennici 50: 95-98.

Newman M., Ketphanh S., Svengsuksa B., Thomas P., Sengdala K., Lamxay V., Armstrong K. 2007. A checklist of the vascular plants of Lao PDR. Royal Botanic Garden Edinburgh, Edinburgh, 394 pp.

Pearce N. P., Cribb P. J. 2002. The orchids of Bhutan. Royal Botanic Garden Edinburgh, Royal Government of Bhutan, Huddersfield, 643 pp.

Schuiteman A., Averyanov L., Rybkova R. 2013. Vanilla atropogon, a new species from Vietnam. Orchideen Journal 1(1): 10-16.

Schuiteman A., Bonnet P, Svengsuksa B., Barthelemy D. 2008. An annotated checklist of the Orchidaceae of Laos. Nordic Journal of Botany 26: 257-316. DOI: 10.1111/j.1756-1051.2008.00265.x

Seidenfaden G. 1976. Orchid genera in Thailand. IV. Liparis L. C. Rich. Dansk Botanisk Arkiv 31(1): 1-105.

Tetsana N., Pedersen H.A., Sridith K. 2013. Five species of Liparis (Orchidaceae) newly recorded for Thailand. Thai Forest Bulletin (Bot.) 41: 48-55. 